\title{
Uniformly best wavenumber approximations by spatial central difference operators
}

\author{
Viktor Linders ${ }^{\mathrm{a}}$, Jan Nordström ${ }^{\mathrm{b}}$ \\ ${ }^{a}$ Division of Computational Mathematics, Computational Mathematics, Linköping \\ University, SE-581 83 Linköping, Sweden (viktor.linders@liu.se). \\ ${ }^{b}$ Division of Computational Mathematics, Computational Mathematics, Linköping \\ University, SE-581 83 Linköping, Sweden (jan.nordstrom@liu.se).
}

\begin{abstract}
We construct accurate central difference stencils for problems involving high frequency waves or multi-frequency solutions over long time intervals with a relatively coarse spatial mesh, and with an easily obtained bound on the dispersion error. This is done by demonstrating that the problem of constructing central difference stencils that have minimal dispersion error in the infinity norm can be recast into a problem of approximating a continuous function from a finite dimensional subspace with a basis forming a Chebyshev set. In this new formulation, characterising and numerically obtaining optimised schemes can be done using established theory.
\end{abstract}

Keywords: Dispersion relation; Wave propagation; Wavenumber approximation; Finite differences; Approximation theory

\section{Introduction and motivation}

Consider an approximation of a first derivative $u_{x}$ of some function $u(x, t)$ at the point $x=x_{i}$. Finite difference stencils have traditionally been constructed by optimising the approximation accuracy with respect to the bandwidth of the scheme. For central stencils using $p$ values of $u$ on either side of $x_{i}$, this is normally done using Taylor expansions with the requirement that

$$
\left(\frac{\partial u}{\partial x}\right)_{i}+\mathcal{O}\left(\Delta x^{2 p}\right)=\frac{1}{\Delta x} \sum_{k=1}^{p} c_{k}^{(p)}\left(u_{i+k}-u_{i-k}\right),
$$


which uniquely determines the stencil coefficients $c_{k}^{(p)}$. Here $\Delta x$ is the spatial step size of the discretisation. Throughout this paper we will refer to such schemes as classical and quantities related to these schemes will be denoted with a subscript $c$.

The philosophy behind the classical stencils is that the error in the approximation is dominated by the truncation error of the corresponding Taylor series. However, for problems involving the propagation of high frequency waves over large intervals the error may instead be dominated by inexact approximations of the dispersion relation. Such waves require small spatial increments in order to be properly resolved, which puts restrictions on $\Delta x$.

Problems of this type are common in computational fluid dynamics, aeroacoustics, electromagnetism, elasticity and seismology, see e.g. [1, 2, 3, 4, 5, 6]. Wave properties encoded within the dispersion relation include phase velocity, group velocity, anisotropy and dissipation. It is therefore of interest to develop difference schemes that preserve the analytic dispersion relation of the governing equations for a wide range of spatial increments.

Such schemes have received considerable attention during the past few decades. Among the earliest comprehensive treatments are [7, 8]. Largely, these schemes may be divided in two categories: Those where the spatial and temporal discretisations are simultaneously optimised in order to preserve the dispersion properties, and those where they are kept separate. Examples of schemes belonging to the former category were presented in $[9,10]$. In the latter category, early compact stencils were presented in [11] and improved in [12]. Non-compact stencils, which is the main focus of this paper, have been derived in $[13,14,15,16]$. Some of these will later be used for comparison. For other approaches, see e.g. [17, 18, 19, 20, 21, 22]. A comparison of many of these schemes was presented in [23].

In this paper we will construct new central difference stencils that approximate first derivatives. These schemes have suboptimal accuracy relative to the bandwidth, leaving a set of free stencil parameters that will be used to preserve the dispersion relation. We will minimise the dispersion error uniformly, that is in the $L^{\infty}$-sense, which results in stencils with an easily computable, guaranteed largest dispersion error for any wavenumber in some 
finite range.

This paper is structured as follows: In Section 2 we give some preliminary information regarding notation and relevant theoretical concepts. In Section 3 we discuss dispersion properties of the classical central difference stencils. In Section 4 we formulate and solve the problem of constructing stencils with uniformly minimised dispersion error. In Section 5 we give some details about how such schemes are found numerically. We discuss boundary treatments using Summation-by-parts operators in Section 6. The theoretical findings are corroborated with numerical examples in Section 7, where comparisons are made with other schemes presented in the literature. We finish with concluding remarks in Section 8.

\section{Preliminaries}

Before we proceed we introduce the notation and theoretical concepts that we will require.

\subsection{Notation}

Throughout this paper we will separate analytic quantities, quantities relating to classical central difference schemes (denoted with a subscript $c$ ), and quantities relating to new, optimised schemes. Approximations are marked with an overbar. In Table 1 we list those quantities where confusion may occur.

\begin{tabular}{|l|l|l|l|}
\hline & Analytic & Classical & Optimised \\
\hline Frequency & $\omega$ & $\bar{\omega}_{c}$ & $\bar{\omega}$ \\
Wavenumber & $\kappa$ & $\bar{\kappa}_{c}$ & $\bar{\kappa}$ \\
Normalised wavenumber & $\xi=\kappa \Delta x$ & $\bar{\xi}_{c}$ & $\bar{\xi}$ \\
Phase speed & $v_{p}$ & $\bar{v}_{p c}$ & $\bar{v}_{p}$ \\
Group speed & $v_{g}$ & $\bar{v}_{g c}$ & $\bar{v}_{g}$ \\
Dispersion error & & $E_{c}(\xi)=\xi-\bar{\xi}_{c}$ & $E(\xi, \mathbf{a})=\xi-\bar{\xi}$ \\
\hline
\end{tabular}

Table 1: Notation

Functions will be denoted by lower case letters, e.g. $u(x, t)$, unless otherwise specified. If a function is approximated on a discrete grid we write the approximation as a vector, e.g. $\mathbf{u}=\left(u_{0}, \ldots u_{N}\right)^{T}$. Here $u_{j}$ is the $j^{\text {th }}$ element 
of the $(N+1)$-dimensional vector $\mathbf{u}(t)$ approximating the function $u(x, t)$ at the point $x=x_{j}, 0 \leq j \leq N$. Let $\Delta x=x_{j+1}-x_{j}$ be the spatial step size. Then $x_{j}=j \Delta x$.

\subsection{Analytic and numerical dispersion relations}

In this paper we consider the numerical dispersion relations of explicit central difference stencils approximating a first derivative in space. We will therefore study semi-discretisations with no concern for the role of the time dependence. As our starting point it is thus sufficient to consider a governing partial differential equation with a single first derivative in space. The simplest example is the periodic advection equation,

$$
u_{t}+c u_{x}=0, \quad 0 \leq t
$$

where we assume that $c>0$. Assume further that the solution $u(x, t)$ has a uniformly convergent Fourier expansion. Then we may consider solutions in the form of a general Fourier mode, $u(x, t)=\exp (i(\kappa x-\omega t))$, with wavenumber $\kappa$ and angular frequency $\omega$. Inserting $u$ into (2) implies

$$
\omega=c \kappa .
$$

Phase speed $v_{p}$, and group speed $v_{g}$, are respectively defined as

$$
v_{p}=\frac{\omega}{\kappa}=c, \quad v_{g}=\frac{\partial \omega}{\partial \kappa}=c .
$$

Thus (2) describes a wave travelling to the right at speed $c$. The relation (3) is the analytic dispersion relation. The problem (2) is non-dispersive in the sense that the phase speed is independent of the wavenumber, i.e. all waves of any frequency propagate equally fast.

Let us discretise (2) with a classical spatial central difference operator $D$ of the form (1);

$$
\frac{\mathrm{d} u_{j}}{\mathrm{~d} t}+c D u_{j}=0
$$

where $u_{j}$ approximates $u\left(x_{j}, t\right)$. Again, inserting a general Fourier mode gives the numerical dispersion relation

$$
\bar{\omega}_{c}=2 \frac{c}{\Delta x} \sum_{k=1}^{p} c_{k}^{(p)} \sin (k \Delta x \kappa)
$$


where $c_{k}^{(p)}, k=1, \ldots, p$ as before are the stencil coefficients of the $2 p^{\text {th }}$ order classical scheme. Note that we have introduced an overbar to signify that we are considering a numerical approximation of the analytic frequency. The numerical phase and group speeds of the classical scheme are

$$
\bar{v}_{p c}=\frac{\bar{\omega}_{c}}{\kappa}, \quad \bar{v}_{g c}=\frac{\partial \bar{\omega}_{c}}{\partial \kappa} .
$$

Note from (4) and (5) that the numerical phase speed is dependent on the wavenumber, $\kappa$. The approximation is thus inherently dispersive, which is what gives rise to the dispersion error and subsequent errors in phase and group velocity etc.

We let $\xi=\kappa \Delta x$ be the normalised wavenumber and subsequently normalise (4) with $\Delta x / c$ :

$$
\bar{\xi}_{c} \equiv \bar{\kappa}_{c} \Delta x=\frac{\bar{\omega}_{c} \Delta x}{c}=2 \sum_{k=1}^{p} c_{k}^{(p)} \sin (k \xi) .
$$

Here we have simply defined $\bar{\kappa}_{c}=\bar{\omega}_{c} / c$ in analogy with (3).

Noting that the smallest wavelength the scheme can resolve is $\lambda_{\min }=2 \Delta x$, we find that the largest resolvable wavenumber is $\kappa_{\max }=2 \pi / \lambda_{\min }=\pi / \Delta x$. Thus we always have $|\xi| \leq \pi$, though typically we will consider some smaller range of wavenumbers, $\xi \in\left[0, \xi_{\max }\right]$.

Equation (6) gives the (normalised) numerical wavenumber as a function of the analytic wavenumber, $\xi \in[0, \pi]$. We define the dispersion error of the classical stencil as $E_{c}(\xi)=\xi-\bar{\xi}_{c}$. Note from (5) and (6) that

$$
\bar{\xi}_{c}=\frac{\bar{\omega}}{c} \Delta x=\frac{\kappa \Delta x}{c} \bar{v}_{p}=\frac{\xi}{c} \bar{v}_{p}
$$

Thus

$$
\xi-\bar{\xi}_{c}=\frac{\xi}{c}\left(c-\bar{v}_{p}\right)=\frac{\xi}{c}\left(v_{p}-\bar{v}_{p}\right),
$$

so the dispersion error, $E_{c}(\xi)$ is just a rescaling of the error in the phase speed. It should be noted that a study of the error in the group speed would also be of interest, however, our focus will be on functions of the same type as (7). 


\subsection{Approximation theory}

Let $g(x), \theta_{i}(x), i=1, \ldots, n$ be continuous functions of $x \in\left[x_{L}, x_{R}\right]$. In this paper we will face problems of the form

$$
\text { find } \underset{\mathbf{a} \in \mathbb{R}^{n}}{\operatorname{argmin}}\|r(x, \mathbf{a})\|_{\infty},
$$

where

$$
r(x, \mathbf{a})=g(x)-\sum_{i=1}^{n} a_{i} \theta_{i}(x)
$$

and $\mathbf{a}=\left(a_{1}, \ldots, a_{n}\right)$. Without loss of generality we may assume that the set $\left\{\theta_{i}\right\}$ is linearly independent (for otherwise we could consider some smaller linearly independent subset), that is $\sum a_{i} \theta_{i}(x)=0$ if and only if $\mathbf{a}=\mathbf{0}$. Thus the functions $\theta_{i}$ form a basis for some $n$-dimensional vector space. The problem (8) is therefore about approximating a continuous function from a subspace of finite dimension.

It is of course desirable to know if a solution to (8) exists and, if so, whether or not it is unique. To answer these questions we need the following definitions (see e.g. [24] for details):

Definition 1. The set of functions, $\left\{\theta_{i}\right\}, i=1, \ldots, n$ forms a Chebyshev set on the interval $\left[x_{L}, x_{R}\right]$ if any non-trivial linear combination has at most $(n-1)$ zeros in in this interval.

Definition 2. Let $M(\mathbf{a})$ denote the set of points $\left\{x_{1}, x_{2}, \ldots\right\}$ at which $|r(x, \mathbf{a})|=$ $\|r(x, \mathbf{a})\|_{\infty}$. Then $r(x, \mathbf{a})$ is said to alternate $n$ times on $\left[0, \xi_{\max }\right]$ if $M(\mathbf{a})$ contains $(n+1)$ points $x_{L} \leq x_{1}<x_{2}<\cdots<x_{n+1} \leq x_{R}$ such that

$$
r\left(x_{i}, \mathbf{a}\right)=-r\left(x_{i+1}, \mathbf{a}\right), \quad i=1, \ldots, n .
$$

The set $\left\{x_{i}\right\}$ is referred to as an alternating set.

The following result due to Young [25] answers the questions of existence and uniqueness of a solution to the problem (8):

Theorem 1. Let $\left\{\theta_{i}\right\}, i=1, \ldots, n$ be a Chebyshev set on $\left[x_{L}, x_{R}\right]$. Then there exists a unique solution a to problem (8). For this solution, $r(x, \mathbf{a})$ has an alternating set of $(n+1)$ points in $\left[x_{L}, x_{R}\right]$.

For convenience we will also use the following definition:

Definition 3. If a vector a solves problem (8), then $\sum a_{i} \theta_{i}$ is called a best approximation of $g(x)$. 


\section{Classical central difference stencils}

We begin with a discussion of why classical schemes are non-optimal for wavenumber approximations. Recall that a subscript $c$ denotes quantities related to the classical stencils.

Consider again the $(2 p+1)$ point classical central difference stencil of order $\mathcal{O}\left(\Delta x^{2 p}\right)$ in $(1)$. As before, $c_{k}^{(p)}$ are the stencil coefficients of the $2 p^{\text {th }}$ order classical scheme. A Taylor expansion reveals that in order to achieve desired accuracy, $c_{k}^{(p)}$ must for all $k$ satisfy

$$
\left(\begin{array}{cccc}
1 & 2 & \ldots & p \\
1 & 2^{3} & \ldots & p^{3} \\
\vdots & \vdots & & \vdots \\
1 & 2^{2 p-1} & \ldots & p^{2 p-1}
\end{array}\right)\left(\begin{array}{c}
c_{1}^{(p)} \\
c_{2}^{(p)} \\
\vdots \\
c_{p}^{(p)}
\end{array}\right)=\left(\begin{array}{c}
\frac{1}{2} \\
0 \\
\vdots \\
0
\end{array}\right) .
$$

The solution to this system of equations is known to be (see e.g. [26])

$$
c_{k}^{(p)}=(-1)^{k-1} \frac{\left(\begin{array}{c}
p \\
k
\end{array}\right)}{k\left(\begin{array}{c}
p+k \\
p
\end{array}\right)}=(-1)^{k-1} \frac{(p !)^{2}}{k(p-k) !(p+k) !} .
$$

The following observation is useful. The proof is found in Appendix A.

Lemma 1. Consider the function

$$
f_{c}^{(p)}(x)=1-2 \sum_{k=1}^{p} c_{k}^{(p)} k T_{k}(x)
$$

where $T_{k}(x)$ is the $k^{\text {th }}$ order Chebyshev polynomial of the first kind, uniquely defined through the relation $T_{k}(\cos (\phi))=\cos (k \phi)$. Then

$$
f_{c}^{(p)}(x)=d_{p}(1-x)^{p}, \quad d_{p}=\frac{2^{p}}{\left(\begin{array}{c}
2 p \\
p
\end{array}\right)} .
$$

We are in position to show

Theorem 2. Classical central difference stencils underestimate the phase and group speeds of propagating solutions. 
Proof. From (6) we have that

$$
\bar{\xi}_{c}=2 \sum_{k=1}^{p} c_{k}^{(p)} \sin (k \xi)
$$

is the numerical wavenumber associated with a classical stencil. We define the corresponding error function

$$
E_{c}(\xi):=\xi-\bar{\xi}_{c}=\xi-2 \sum_{k=1}^{p} c_{k}^{(p)} \sin (k \xi) .
$$

Let $x=\cos (\xi)$. Then

$$
\begin{aligned}
\frac{\mathrm{d} E_{c}}{\mathrm{~d} \xi} & =1-2 \sum_{k=1}^{p} c_{k}^{(p)} k \cos (k \xi)=1-2 \sum_{k=1}^{p} c_{k}^{(p)} k T_{k}(\cos (\xi)) \\
& =d_{p}(1-\cos (\xi))^{p}=f_{c}^{(p)}(\cos (\xi)) .
\end{aligned}
$$

Note that $\frac{\mathrm{d} E_{c}}{\mathrm{~d} \xi}=f_{c}^{(p)}(\cos (\xi))=0$ only when $\cos (\xi)=1$, i.e. $E_{c}$ has extrema only at $\xi=2 \pi n, n=0, \pm 1, \ldots$ However, since $\xi \in\left[0, \xi_{\max }\right] \subseteq[0, \pi]$ it is clear that $E_{c}(\xi)$ has an extreme point at $\xi=0$ and no other extrema in the domain of interest. Consequently $E_{c}(\xi)$ is monotonic for $\xi \in[0, \pi]$.

Note also that $\frac{\partial E_{c}}{\partial \xi} \geq 0$, indicating that $E_{c}(\xi)$ is increasing. We thus have $\bar{\xi}_{c} \leq \xi$ with equality only at $\xi=0$. From (6) we therefore have $\bar{\omega}_{c} \leq \omega$ where as before $\bar{\omega}_{c}$ is the numerical frequency. It follows from (5) that the numerical wavespeed satisfies

$$
\bar{v}_{p c}=\frac{\bar{\omega}_{c}}{\kappa} \leq \frac{\omega}{\kappa}=v_{p}
$$

where equality holds only when $\xi=0$.

Next note from the definitions of $E_{c}$ and $\xi$ that the analytic and numerical group speeds are related by

$$
0 \leq \frac{\partial E_{c}}{\partial \xi}=\frac{\Delta x}{c} \frac{\partial \kappa}{\partial \xi} \frac{\partial}{\partial \kappa}\left(\omega-\bar{\omega}_{c}\right)=\frac{1}{c}\left(v_{g}-\bar{v}_{g c}\right),
$$

where in the first inequality we have used the fact that $f_{c}^{(p)}(\cos (\xi)) \geq 0$. Since $c>0$ by assumption we have $\bar{v}_{g c} \leq v_{g}$ with equality only when $\xi=0$. 
Remark 1. Since $E_{c}(\xi)$ is monotonically increasing it is clear that the largest error will occur at the highest wavenumber. It is therefore easy to write down an expression for $\left\|E_{c}\right\|_{\infty}$. We have

$$
\begin{aligned}
\left\|E_{c}\right\|_{\infty} & =E_{c}\left(\xi_{\max }\right)=\int_{0}^{\xi_{\max }} \frac{d E_{c}}{d \xi} d \xi \\
& =\int_{0}^{\xi_{\max }} f_{c}^{(p)}(\cos (\xi)) d \xi=\frac{2^{p}}{\left(\begin{array}{c}
2 p \\
p
\end{array}\right)} \int_{0}^{\xi_{\max }}(1-\cos (\xi))^{p} d \xi
\end{aligned}
$$

where we have used the fact that $E_{c}(0)=0$. Note that for $\xi>\pi / 2$ the integrand, and thus the error, starts to grow rapidly with increasing $p$. Thus, even very high order classical stencils fail for wavenumbers above this limit.

The problem with the classical schemes lies in the monotonicity of the error function. In the following sections we will remedy this by considering wider stencils with reduced formal accuracy but with a set of free parameters that we use to minimise the dispersion error.

\section{Improved numerical dispersion relations}

In view of the above analysis it is desirable to modify the stencil (6) in a way that makes the numerical wavenumber an as good approximation of $\xi$ as possible. We will briefly discuss previous approaches to this optimisation problem, but our main focus will be on uniformly best schemes, i.e. optimised in the $L^{\infty}$-norm.

\subsection{Other approaches}

A straightforward option introduced by Tam and Webb [13] is to sacrifice the optimal order of accuracy of the difference operator and use the resulting free parameter to minimise the error function defined in the $L^{2}$-norm as

$$
E_{D R P}:=\int_{\Omega}|\xi-\bar{\xi}|^{2} \mathrm{~d} \xi
$$

where the integral runs over some continuous spatial domain $\Omega$. In [13] the chosen domain is $\xi \in[0, \pi / 2]$ and a seven point wide, fourth order accurate stencil is presented. These types of schemes are called "Dispersion Relation Preserving" (DRP). Here we will use a similar DRP scheme, though optimised for $\xi \in[0, \pi / 3]$, for comparison with the new stencils we shall obtain 
later. We will henceforth denote this stencil $\operatorname{DRP}(4,7)$.

An alternative to the DRP procedure is considered by Bogey and Bailly [16] where the error is defined as

$$
E_{B B}:=\int_{\log (\xi)_{L}}^{\log (\xi)_{H}}|\xi-\bar{\xi}| \mathrm{d}(\log (\xi)) .
$$

Several stencils of this type is presented in [16], however we shall for ease of comparison limit our attention to a nine point stencil of fourth order accuracy optimised for wavenumbers $\xi \in[\pi / 16, \pi / 2]$. This stencil will from hereon be denoted $\mathrm{BB}(4,9)$.

Zingg, Lomax and Jurgens [14, 15] present a seven point wide stencil that is developed to uniformly minimise the error for waves resolved with at least ten grid points per wavelength, i.e. $\xi \in[0, \pi / 5]$. Details of how this optimisation is performed are not given. The stencil consists of a second order antisymmetric central difference operator and a first order symmetric filter, which dissipates high wavenumber modes. We will denote this stencil ZLJ $(2,7)$.

We will use the above schemes as comparisons with the new derived subsequently. The coefficients of all stencils used are found in Table C.2 (Appendix C).

\subsection{Uniform minimisation}

We will henceforth consider approximations in the general domain $\xi \in\left[0, \xi_{\text {max }}\right]$ where $0<\xi_{\max }<\pi$. Specifically, we will investigate the properties of wavenumber approximations, $\bar{\xi}$, that minimise

$$
\max _{\xi \in\left[0, \xi_{\max }\right]}|\xi-\bar{\xi}| .
$$

This means that each wavenumber in the domain $\left[0, \xi_{\max }\right]$ is approximated with an error not exceeding some upper bound. For problems involving a range of wavenumbers, perhaps not all known, this is clearly convenient.

Let us now, like for the above schemes, perturb the stencil (1) without increasing the accuracy. However, whereas for the previously introduced schemes a 
given number of free parameters were added to the stencil, we assume the addition of $n \geq 1$ new coefficients. Thus, the stencil we consider has the form

$$
\left(\frac{\partial u}{\partial x}\right)_{i}+\mathcal{O}\left(\Delta x^{2 p}\right)=\frac{1}{\Delta x} \sum_{k=1}^{p+n} a_{k}\left(u_{i+k}-u_{i-k}\right) .
$$

Such a scheme uses $(p+n)$ points on either side of $x_{i}$ to approximate the derivative, however the formal order of accuracy remains $\mathcal{O}\left(\Delta x^{2 p}\right)$. Here $a_{k}, k=1, \ldots, p+n$ are the coefficients of the new stencil.

In view of (6) the numerical wavenumber corresponding to (11) is

$$
\bar{\xi}=2 \sum_{k=1}^{p+n} a_{k} \sin (k \xi) .
$$

Let $\tilde{\mathbf{a}}=\left(a_{1}, \ldots, a_{p+n}\right)$. Our goal may thus be formulated in terms of the following problem:

Find $\underset{\tilde{\mathbf{a}} \in \mathbb{R}^{p+n}}{\operatorname{argmin}}\|E(\xi, \tilde{\mathbf{a}})\|_{\infty} \quad$ ensuring formal accuracy $\mathcal{O}\left(\Delta x^{2 p}\right)$.

Here $E(\xi, \tilde{\mathbf{a}})=\xi-\bar{\xi}$. Clearly this problem is similar to the problem (8). Indeed we are approximating the continuous function $\xi$ from a finite dimensional subspace spanned by the linearly independent set of functions $\{\sin (k \xi)\}, k=1, \ldots, p+n$. However, the accuracy requirement puts constraints on the choice of $\tilde{\mathbf{a}}$ and it is not trivial to tell whether we are dealing with a Chebyshev set or not. It is therefore of interest to reformulate problem (13).

The coefficients $a_{k}$ are subject to the same accuracy constraints as the classical stencils. We therefore have precisely $n$ degrees of freedom and we will, without loss of generality, let these be the $n$ coefficients $a_{p+1}, a_{p+2}, \ldots, a_{p+n}$. The coefficients must, as before, satisfy

$$
\left(\begin{array}{cccccc}
1 & 2 & \ldots & p & \ldots & (p+n) \\
1 & 2^{3} & \ldots & p^{3} & \ldots & (p+n)^{3} \\
\vdots & \vdots & & \vdots & & \vdots \\
1 & 2^{2 p-1} & \ldots & p^{2 p-1} & \ldots & (p+n)^{2 p-1}
\end{array}\right)\left(\begin{array}{c}
a_{1} \\
a_{2} \\
\vdots \\
a_{p} \\
\vdots \\
a_{p+n}
\end{array}\right)=\left(\begin{array}{c}
\frac{1}{2} \\
0 \\
\vdots \\
0
\end{array}\right)
$$


Equivalently we may write

$$
\begin{aligned}
&\left(\begin{array}{cccc}
1 & 2 & \ldots & p \\
1 & 2^{3} & \ldots & p^{3} \\
\vdots & \vdots & & \vdots \\
1 & 2^{2 p-1} & \ldots & p^{2 p-1}
\end{array}\right)\left(\begin{array}{c}
a_{1} \\
a_{2} \\
\vdots \\
a_{p}
\end{array}\right) \\
&=\left(\begin{array}{c}
\frac{1}{2} \\
0 \\
\vdots \\
0
\end{array}\right)-\left(\begin{array}{c}
(p+1) \\
(p+1)^{3} \\
\vdots \\
(p+1)^{2 p-1}
\end{array}\right) a_{p+1}-\ldots-\left(\begin{array}{c}
(p+n) \\
(p+n)^{3} \\
\vdots \\
(p+n)^{2 p-1}
\end{array}\right) a_{p+n} .
\end{aligned}
$$

Clearly each $a_{k}, k=1, \ldots, p$ is linearly dependent on each $a_{p+j}, j=1, \ldots, n$. Note in particular that if $a_{p+j}=0, \forall j \geq 1$, the solution reduces to $a_{k}=$ $c_{k}^{(p)}, k=1, \ldots, p$. We may thus write

$$
a_{k}=c_{k}^{(p)}+\sum_{j=1}^{n} \alpha_{k}^{(j)} a_{p+j}
$$

where the parameters $\alpha_{k}^{(j)}, k=1, \ldots, p$ are independent of $a_{p+j}, j=1, \ldots, n$. Comparing terms multiplying $a_{p+j}$ we find that $\alpha_{k}^{(j)}$ must satisfy

$$
\left(\begin{array}{cccc}
1 & 2 & \ldots & p \\
1 & 2^{3} & \ldots & p^{3} \\
\vdots & \vdots & & \vdots \\
1 & 2^{2 p-1} & \ldots & p^{2 p-1}
\end{array}\right)\left(\begin{array}{c}
\alpha_{1}^{(j)} \\
\alpha_{2}^{(j)} \\
\vdots \\
\alpha_{p}^{(j)}
\end{array}\right)=-\left(\begin{array}{c}
(p+j) \\
(p+j)^{3} \\
\vdots \\
(p+j)^{2 p-1}
\end{array}\right), \quad j=1, \ldots, n .
$$

We summarise in the following proposition:

Proposition 1. The numerical wavenumber of the $2 p^{\text {th }}$ order central difference stencil (11), i.e.

$$
\bar{\xi}=2 \sum_{k=1}^{p+n} a_{k} \sin (k \xi),
$$

can be equivalently written as

$$
\bar{\xi}=\bar{\xi}_{c}+\psi(\xi, \mathbf{a})
$$


where $\bar{\xi}_{c}$ is the wavenumber approximation of the $2 p^{\text {th }}$ order classical central difference stencil and

$$
\psi(\xi, \mathbf{a})=\sum_{j=1}^{n} a_{p+j} \phi_{j}(\xi), \quad \phi_{j}(\xi)=2 \sin ((p+j) \xi)+2 \sum_{k=1}^{p} \alpha_{k}^{(j)} \sin (k \xi) .
$$

Here, $\mathbf{a}=\left(a_{p+1}, \ldots, a_{p+n}\right)$ is a vector containing the free stencil parameters. The coefficients $\alpha_{k}^{(j)}$ solve (15) for all $k=1, \ldots, p$ and $j=1, \ldots, n$.

Proof. Inserting (14) into (12) and collecting terms multiplying $a_{p+j}$ gives the desired result.

Note that the set of functions $\left\{\phi_{j}\right\}, j=1, \ldots, n$ is linearly independent for any $n$. Thus the $\phi_{j}$ form a basis for some $n$-dimensional vector space, which we will denote by $\Xi_{n}$.

By Proposition 1 it is clear that the problem (13) may equivalently be written

$$
\text { Find } \underset{\mathbf{a} \in \mathbb{R}^{p+n}}{\operatorname{argmin}}\|E(\xi, \mathbf{a})\|_{\infty}=\left\|E_{c}(\xi)-\psi(\xi, \mathbf{a})\right\|_{\infty}
$$

where $E_{c}(\xi)=\xi-\bar{\xi}_{c}$ is the dispersion error of the classical stencil and $\psi(\xi, \mathbf{a}) \in \Xi_{n}$.

Problem (16) is precisely of the type (8) and thus a unique solution is guaranteed to exists and can be characterised if $\left\{\phi_{j}\right\}, j=1, \ldots, n$ forms a Chebyshev set. We can show

Theorem 3. The set $\left\{\phi_{j}\right\}, j=1, \ldots, n$ defined in Proposition 1 forms a Chebyshev set on the semi-open interval $\left(0, \xi_{\max }\right]$.

The proof is found in Appendix B. The exclusion of the point $\xi=0$ causes no issue. In fact, we have

Corollary 1. Let $\left\{\phi_{j}(\xi)\right\}, j=1, \ldots, n$ be defined as in Proposition 1. Then a solves problem (16) if and only if there exists an alternating set of $(n+1)$ points in $\left(0, \xi_{\max }\right]$.

Proof. Theorem 3 shows that for any $\delta$ satisfying $0<\delta<\xi_{\max },\left\{\phi_{j}\right\}$ is a Chebyshev set on $\left[\delta, \xi_{\text {max }}\right]$. Thus Theorem 1 applies. Letting $\delta \rightarrow 0$ and observing that at $\xi=0$ the approximation is exact yields the desired result. 


\section{Remez multiple exchange algorithm}

Conveniently, due to Remez $[27,28]$ there is an algorithm that solves problem (8) and thus problems (16) and (13). The Remez multiple exchange algorithm is an iterative procedure that utilises the characterisation theorem (Theorem 1) to find a best solution. This is accomplished by searching for a set of points at which the approximation error forms an alternating set. Briefly the algorithm works as follows:

1. Choose a set $X$ containing $n+1$ points $\xi_{i}$ such that $0<\xi_{1}<\xi_{2}<$ $\cdots<\xi_{n+1} \leq \xi_{\max }$

2. Solve the system

$$
E_{c}\left(\xi_{i}\right)-\psi\left(\xi_{i}, \mathbf{a}\right)=(-1)^{i} e, \quad i=1, \ldots, n+1
$$

for a and $e$.

3. Locate the set $M$ of extreme points of the error curve, $E_{c}-\psi$.

4. If the errors at the points in $M$ form an alternating set, then $\psi(\xi, \mathbf{a})$ is the best uniform wavenumber approximation according to Theorem 1. Otherwise, replace $X$ with $M$ and repeat steps 2 and 3 .

It is known that the algorithm converges for any initial choice of $X$ [29]. Further, since $E_{c}(\xi)$ and the basis functions $\phi_{j}(\xi)$ are all twice continuously differentiable it can also be shown that the convergence is quadratic [30]. Note that the algorithm finds not only the coefficients a but also the error of the approximation, $e$, since $|e| \rightarrow\|E\|_{\infty}$ as the algorithm converges.

Naturally the time it takes for the algorithm to converge depends on the initial choice of the set $X$. A suitable option is $\xi_{i}=\xi_{\max } \sin \left(i \frac{\xi_{\max }}{N+1}\right), i=$ $1, \ldots, N+1$, which, for the stencils considered presently converges in less than ten iterations.

Figure 1 shows a toy example for the error of a stencil optimised using the Remez algorithm. The stencil is second order accurate (i.e. $p=1$ ) and is 31 points wide $(n=14)$. Here we have set $\xi_{\max }=\pi / 2$. Thus, the dispersion error for any numerical wavenumber $0 \leq \xi \leq \pi / 2$ is sharply bounded from above by $\|E\|_{\infty} \sim \mathcal{O}\left(10^{-12}\right)$. Note that the error oscillates $n+1=15$ times as expected from Corollary 1. Stencils of this width are of course not used in practice. 


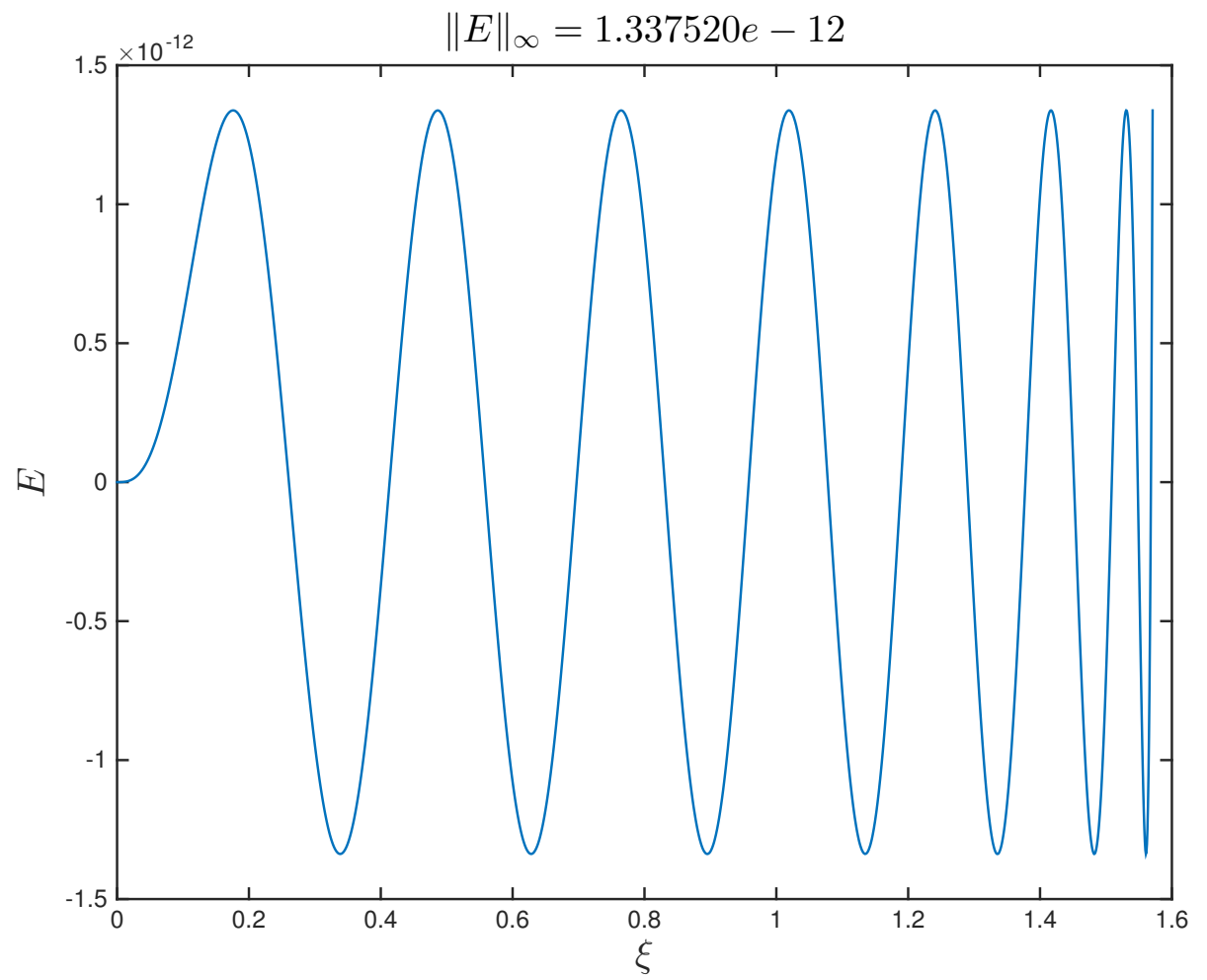

Figure 1: Toy example of a central difference stencil whose coefficients are a solution to problem (16). Here, $p=1$ and $n=14$, i.e. the stencil has formal accuracy $\mathcal{O}\left(\Delta x^{2}\right)$ and is 31 points wide.

\section{A note on boundary treatments}

Naturally it is desirable to extend the above results to domain boundaries where central differences are not easily implemented. One way of doing this is with the use of Summation-By-Parts (SBP) operators augmented with Simultaneous Approximation Terms (SAT) that weakly enforce boundary conditions. The SBP-SAT technique allows for stable and high order discretisations of systems of partial differential equations. For reviews of the SBP-SAT technique and historical notes, see $[31,32]$ and the references therein.

An SBP-operator is defined through the relations

$$
\frac{\partial u}{\partial x} \approx P^{-1} Q \mathbf{u}, \quad P=P^{T}>0, \quad Q+Q^{T}=\mathbf{e}_{\mathbf{N}} \mathbf{e}_{\mathbf{N}}^{T}-\mathbf{e}_{\mathbf{0}} \mathbf{e}_{\mathbf{0}}^{T}
$$


where $P$ and $Q$ are matrices of dimension $(N+1) \times(N+1)$ and $\mathbf{e}_{\mathbf{0}}$ and $\mathbf{e}_{\mathbf{N}}$ are the first and last unit vectors in the standard basis. We will assume that $P$ is a diagonal matrix. As an example of the typical structure of $Q$ we consider

$$
Q=\left(\begin{array}{ccccccc}
-1 / 2 & q_{11} & q_{12} & q_{13} & & & \\
-q_{11} & 0 & q_{21} & q_{22} & & & \\
-q_{12} & -q_{21} & 0 & q_{31} & a_{2} & & \\
-q_{13} & -q_{22} & -q_{31} & 0 & a_{1} & a_{2} & \\
& & -a_{2} & -a_{1} & 0 & a_{1} & a_{2} \\
& & & & & \ddots &
\end{array}\right)
$$

where $a_{1}$ and $a_{2}$ are coefficients of a central difference stencil such as the ones discussed in the previous sections, and $q_{i j}$ are stencil coefficients to be determined.

Note that each row of the block boundary closure of $Q$ consists of a noncentral finite difference stencil, each associated with a corresponding numerical wavenumber and dispersion error. These errors will generally be different. Due to the skew-symmetry of $Q$ we must optimise all rows in the boundary closure simultaneously. A possible strategy, and the first one that we will pursue in a future paper, is to ensure that the largest dispersion error is minimal as measured in the $L^{\infty}$-norm.

\section{Numerical examples}

To illustrate the flexibility of the best wavenumber approximations we present two numerical calculations of different character. The first is a narrow Gaussian pulse solving the one-dimensional advection equation. The second is a vortex solution to the two-dimensional compressible Euler equations. We compare the new stencils (denoted Remez) with those alternatives discussed in section 4.1. The numbers in brackets following a stencil name denote the formal order of accuracy and the width of the stencil respectively.

\subsection{Gaussian pulse}

We consider a profoundly polychromatic solution to the advection equation over the periodic domain $0 \leq x<7$ :

$$
\begin{gathered}
u_{t}+u_{x}=0, \quad 0 \leq x<7, \quad t \geq 0 \\
u(x, 0)=2 \exp \left(-3200(x-1 / 2)^{2}\right) .
\end{gathered}
$$




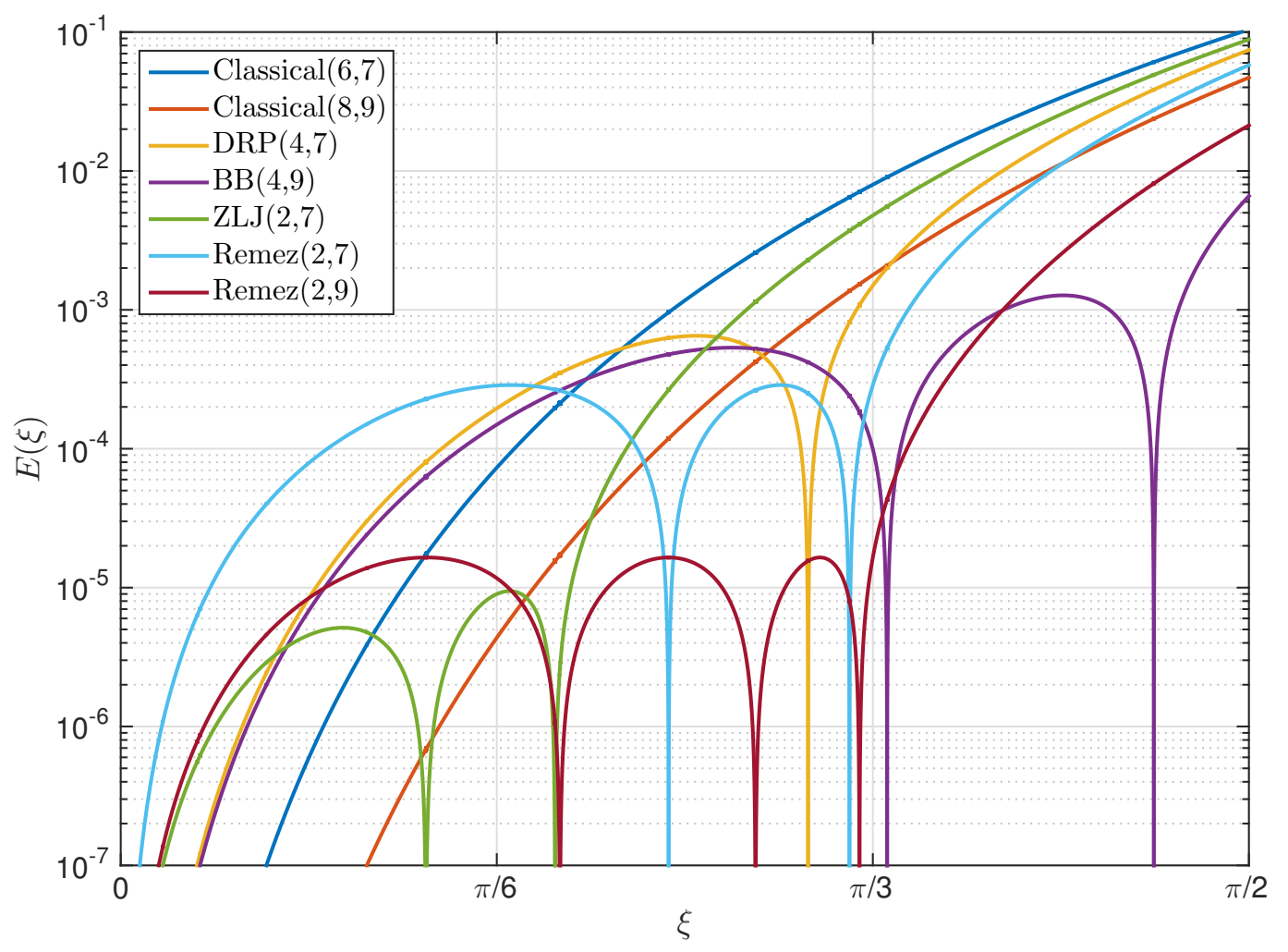

Figure 2: Dispersion errors for the compared schemes.

This pulse is narrow and thus its Fourier transform is wide resulting in a significant contribution from a broad range of wavenumbers. The dominating wavenumber is $\kappa=0$. Contributions from larger wavenumbers decay exponentially but slowly. It makes sense to use a scheme that accurately approximates the dispersion relation near $\xi=\kappa \Delta x=0$ and for some suitably chosen region of larger wavenumbers.

We consider two second order operators (i.e. $p=1$ ) with two and three free parameters $(n=2,3)$ respectively, and optimise these for $\xi \in[0, \pi / 3]$ using the Remez algorithm. The coefficients of the stencils are presented in Appendix C. In Fig. 2 the dispersion errors are shown for the compared schemes. Note how the errors of the two Remez stencils oscillate two and 


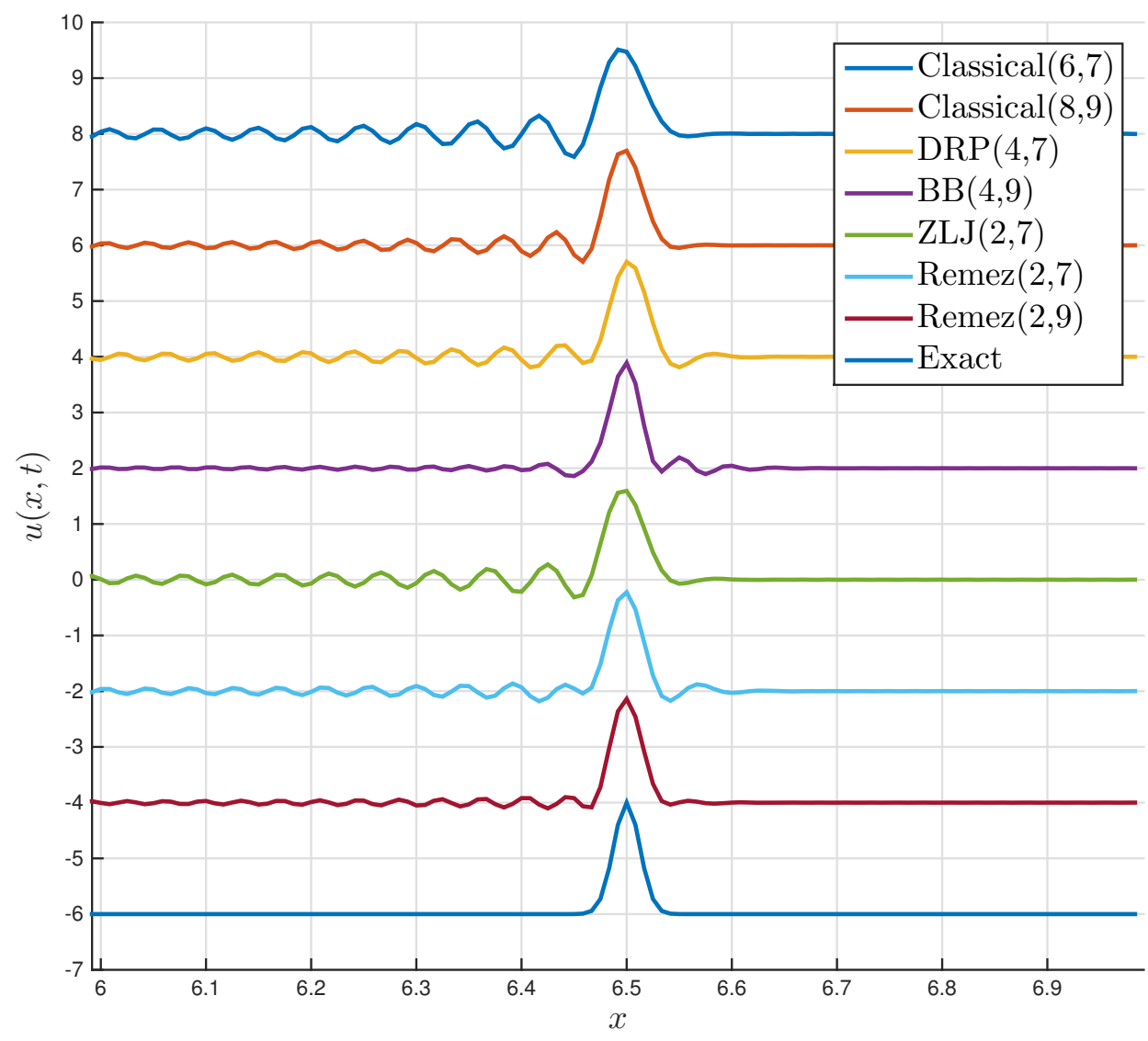

Figure 3: Exact and numerical solutions.

three times respectively, as predicted by Theorem 1 .

We set $\Delta x=1 / 120$ and integrate in time using the classical fourth order Runge-Kutta scheme with time step $\Delta t=10^{-3}$ so the contribution from the temporal discretisation is small. The exact and numerical solutions are shown in Fig. 3, where we have separated the pulses vertically to aid the visualisation. Clearly, all numerical solutions quickly disperse into a train of pulses of decaying amplitude trailing behind the main peak. The pulse trains are a consequence of the inability of the stencils to resolve high frequency 


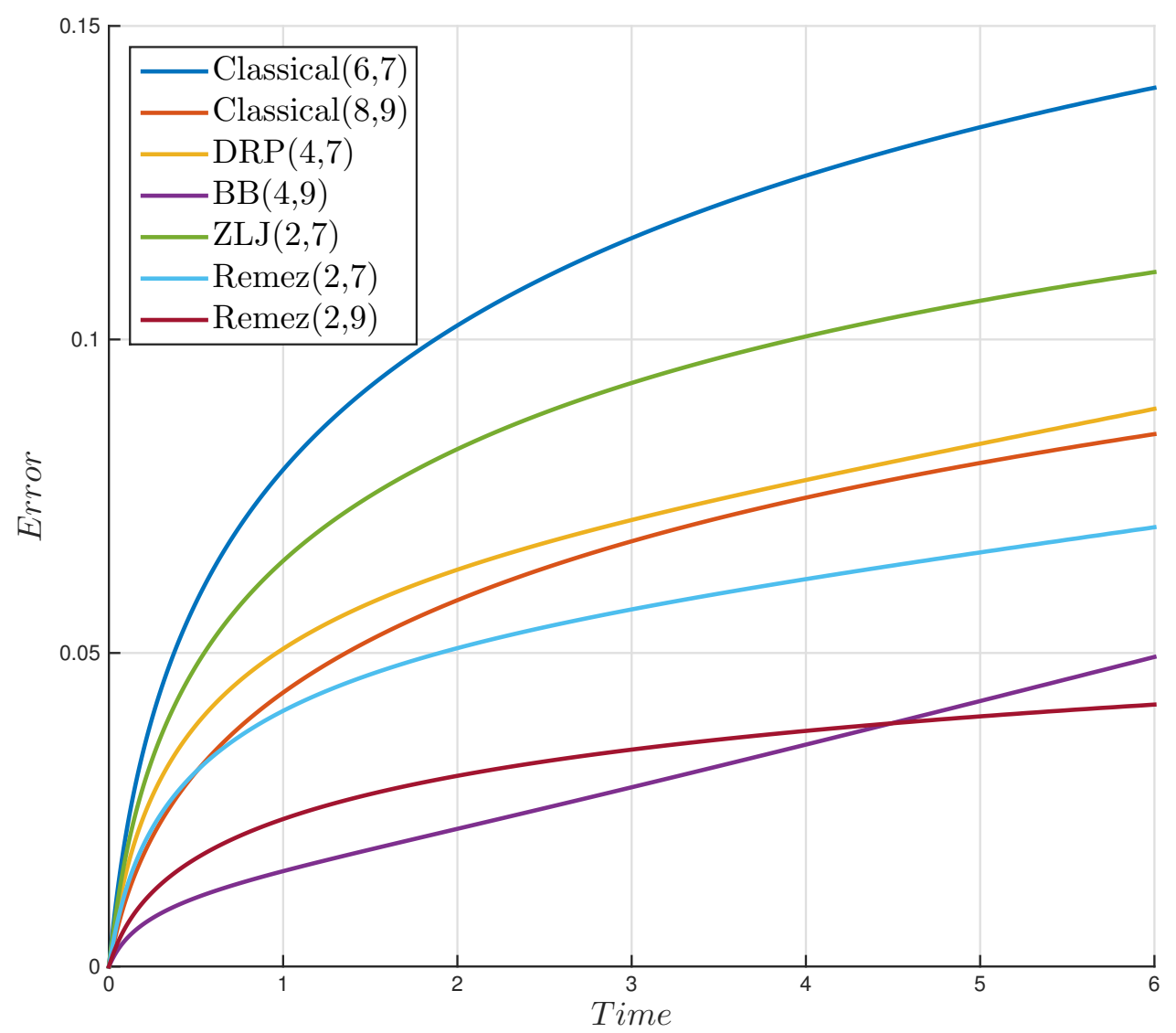

Figure 4: Error development.

Fourier modes properly. The higher frequency modes lag behind more significantly than the lower frequencies as seen from the decaying amplitudes of the trailing pulses. This is as expected from Fig. 2 as the dispersion errors grow rapidly outside their regions of optimisation.

The error development in time is plotted in Fig. 4. The error is here defined as $\|u(x, t)-\mathbf{u}(t)\|_{2}$. Clearly the $\operatorname{Remez}(2,9)$ stencil is the best performer for long times. Note that Remez $(2,7)$ is the best stencil among those that are seven points wide. In fact, it even performs better than the Classical $(8,9)$ 
stencil despite having lower accuracy and utilises less bandwidth.

\subsection{Euler vortex}

The two-dimensional compressible Euler equations can be written in conservative form as

$$
U_{t}+F_{x}+G_{y}=0,
$$

where

$$
U=\left(\begin{array}{c}
\rho \\
\rho u \\
\rho v \\
\rho e
\end{array}\right), \quad F=\left(\begin{array}{c}
\rho u \\
\rho u^{2}+p \\
\rho u v \\
u(e+p)
\end{array}\right), \quad G=\left(\begin{array}{c}
\rho v \\
\rho u v \\
\rho v^{2}+p \\
v(e+p)
\end{array}\right) .
$$

Here $\rho$ is the density; $u$ and $v$ are the velocity components in the $x$-and $y$-directions respectively; $e$ is the total energy density; $p$ is the pressure. The pressure relates to the conservative flow variables in $U$ through the equation of state,

$$
p=(\gamma-1)\left(e-\frac{1}{2} \rho\left(u^{2}+v^{2}\right)\right),
$$

where $\gamma$ is the ratio of specific heats at constant pressure and volume respectively.

We consider the propagation of a vortex along the $x$-axis. The vortex model was introduced in [33] and solves the two-dimensional Euler equations under the assumption of isentropy. The solution can be written

$$
\begin{aligned}
& u=1-\frac{\epsilon y}{2 \pi} \exp \left(\frac{f(x, y, t)}{2}\right) \\
& v=\frac{\epsilon\left(\left(x-x_{0}\right)-t\right)}{2 \pi} \exp \left(\frac{f(x, y, t)}{2}\right) \\
& \rho=\left(1-\frac{\epsilon^{2}(\gamma-1) M^{2}}{8 \pi^{2}} \exp (f(x, y, t))\right)^{\frac{1}{\gamma-1}} \\
& p=\frac{\rho^{\gamma}}{\gamma M^{2}}
\end{aligned}
$$

where $f(x, y, t)=1-\left(\left(\left(x-x_{0}\right)-t\right)^{2}+y^{2}\right) ; \epsilon$ is the circulation; $M$ is the Mach number; $x_{0}$ is the initial position of the vortex on the $x$-axis. 
The analytic solution is used as initial condition for a vortex introduced at $x_{0}=5$ in a periodic domain, $0 \leq x<30,-15 \leq y<15$. We set $M=0.5$ and consider a strong vortex with $\epsilon=5$. We use $\Delta x=\Delta y=1 / 40$ and $\Delta t=1 / 100$. The solution is integrated a total of 1800 time steps to time $t=18$.

We quantify the dispersion error as

$$
E_{\text {disp }}=\left\|(x, y)_{\text {min }}^{a}-(x, y)_{\text {min }}^{n}\right\|_{2},
$$

where $(x, y)_{\text {min }}^{a, n}$ are the locations of the points of minimum pressure of the analytic vortex and numerical approximation respectively. An example is included in Fig. 5. For the approximation, $(x, y)_{\min }^{n}$ is found using the following procedure:

1. Locate the grid point $(x, y)_{m}$ with minimal pressure.

2. Consider the $5 \times 5$-grid consisting of this point and its 24 nearest neighbours, $(x, y)_{m \pm 1,2}$. Use bicubic interpolation to refine the resolution of each point in this sub-grid to $250 \times 250$ points.

3. Locate the point of minimal pressure $(x, y)_{\min }^{n}$ in the refined grid.

4. Repeat 1-3 for each time step.

5. Filter the resulting signal with a boxcar filter of length 200 to reduce noise.

With this procedure the dispersion error becomes a function of wavespeed only, i.e. no amplitude error is considered.

It should be noted that applying central differences to (17) yields an unstable discretisation. Therefore a small amount of artificial dissipation must be added. The dissipation introduces an error in the amplitude of the solution but has no influence on the wave speed and thus does not affect the dispersion error.

We solve (17) using the same seven methods as before. The dispersion errors are shown in Fig. 6. For this particular problem the Remez $(2,9)$ and the $\mathrm{BB}(4,9)$ schemes perform best with very similar error developments whereas Remez $(2,7)$ again is the best among the seven point stencils with an error similar to the classical $8^{\text {th }}$ order stencil. 

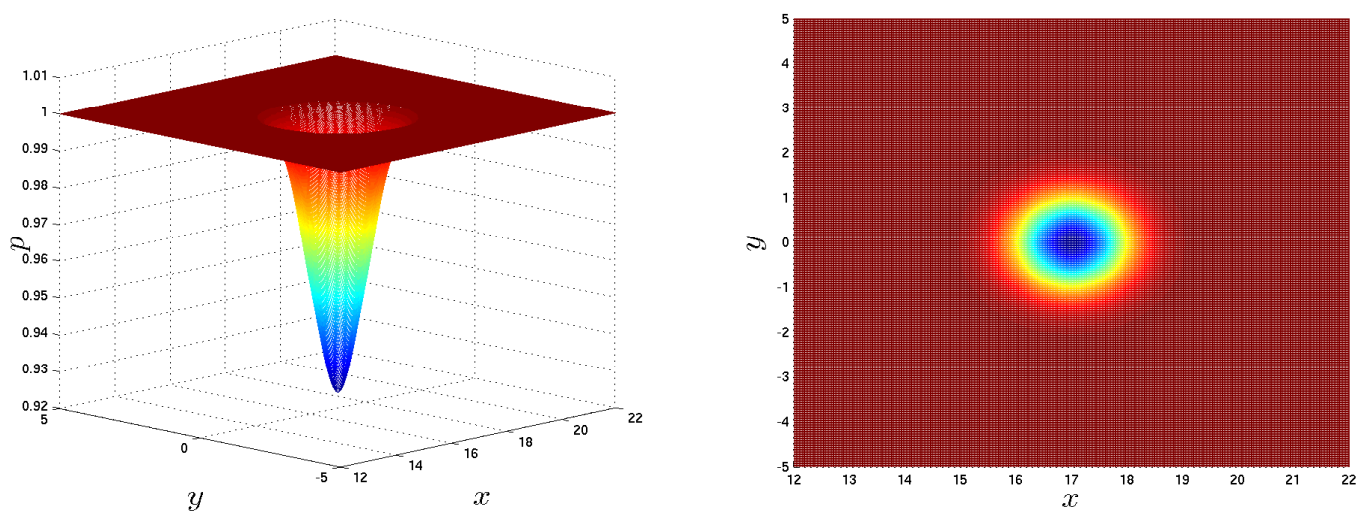

Figure 5: Pressure profile of Euler vortex. The location of the vortex, $(x, y)_{\min }^{a, n}$ is defined as the point of minimum pressure.

\section{Conclusion}

In this paper we have discussed the characterisation and construction of uniformly best wavenumber approximations by central difference schemes. It has been shown that optimising the accuracy of the stencil with respect to the bandwidth (i.e. the classical approach) is not ideal for high-frequency problems over large time intervals. Further it has been shown how this nonoptimality can be understood in terms of the monotonic behaviour of the dispersion error of these classical stencils.

As a suggested remedy we have extended the width of the central difference schemes by introducing an arbitrary number of free parameters without increasing the accuracy. We have seen that the problem of minimising the dispersion error in the $L^{\infty}$-norm can be recast into a problem of approximating the continuous function $E_{c}(\xi)$ by a linear combination of basis functions, $\phi_{j}(\xi)$ from a finite dimensional vector space, $\Xi_{n}$. This new formulation opens the door to using existing approximation theory, and we can immediately state that a unique best solution exists and characterise it in terms of an alternating set. The characterisation subsequently allows for the use of the Remez algorithm to numerically obtain best stencils of desired accuracy and width. As a bonus, the algorithm also provides a sharp error bound for all wavenumbers in the domain of our liking. 


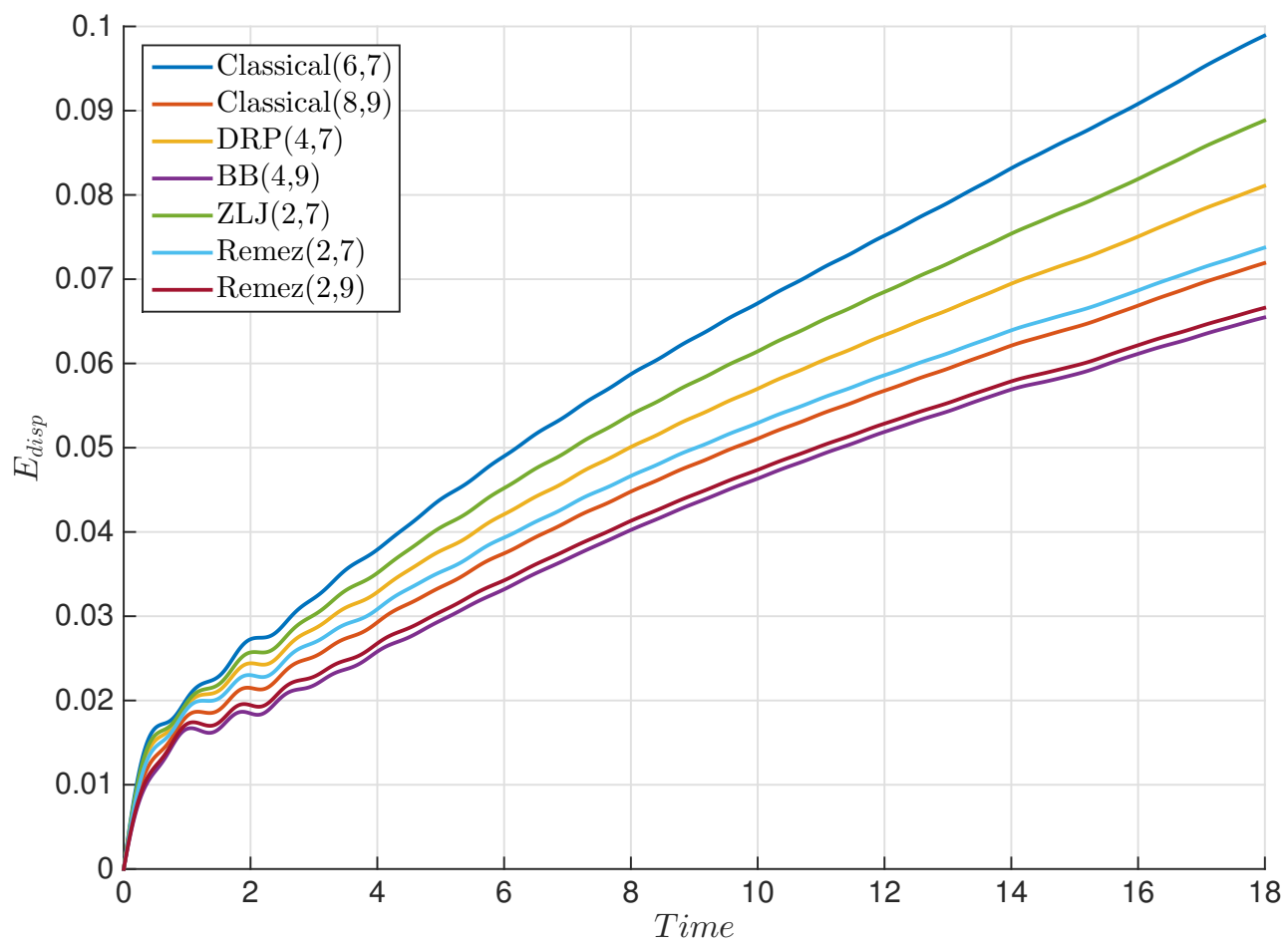

Figure 6: Dispersion error development in time for the compressible Euler equations.

Comparing the new stencils with classical stencils and other schemes presented in the literature we have shown that the Remez stencils are a highly competitive option for accurate wavenumber approximations. Additionally, the construction of such schemes is equally simple for any wavenumber domain of our liking, rendering this approach particularly versatile. This allows for accurate approximations of problems of high frequency waves, or multifrequency solutions, with a relatively coarse spatial mesh.

In conclusion, this new method of constructing schemes of best wavenumber approximation is a flexible technique with applications ranging form computational fluid dynamics and aeroacoustics to electromagnetism and seismology. 


\section{Appendix A. Proof of Lemma 1}

Proof. We need the following well known property of the Chebyshev polynomials:

$$
\left.\frac{\mathrm{d}^{m} T_{k}(x)}{\mathrm{d} x^{m}}\right|_{x=1}= \begin{cases}1 & \text { if } m=0 \\ \prod_{j=0}^{m-1} \frac{k^{2}-j^{2}}{2 j+1} & \text { if } m=1,2, \ldots\end{cases}
$$

We thus have $f_{c}^{(p)}(1)=1-2 \sum_{k=1}^{p} c_{k}^{(p)} k=0$ by the first row of (9). Likewise,

$$
\begin{aligned}
\left.\frac{\mathrm{d}^{m} f_{c}^{(p)}(x)}{\mathrm{d} x^{m}}\right|_{x=1} & =-2 \sum_{k=1}^{p} c_{k}^{(p)} k \prod_{j=0}^{m-1} \frac{k^{2}-j^{2}}{2 j+1} \\
& =\mathcal{O}\left(\sum_{k} c_{k}^{(p)} k^{2 m+1}\right)+\mathcal{O}\left(\sum_{k} c_{k}^{(p)} k^{2 m-1}\right)+\cdots+\mathcal{O}\left(\sum_{k} c_{k}^{(p)} k^{3}\right) .
\end{aligned}
$$

Note that only odd powers of $k$ remain due to the factor $k^{2}$ when $j=0$ and the $k$ in front of the product. Observe that the right hand side is zero whenever $m \leq p-1$, which follows from the remaining rows of (9). Consequently $f_{c}^{(p)}(x)$ has a root of multiplicity $p$ at $x=1$. However, since $f_{c}^{(p)}$ is a polynomial of degree $p$ we may write $f_{c}^{(p)}(x)=d_{p}(1-x)^{p}$ for some constant $d_{p}$ that depends only on $p$. We find $d_{p}$ by writing

$$
\sum_{k=1}^{p} c_{k}^{(p)} k T_{k}(x)=\frac{1}{2}-\frac{d_{p}}{2}(1-x)^{p}
$$

and comparing coefficients in front of the $x^{p}$ term. On the left hand side the $x^{p}$ term occurs only when $k=p$. From (10) we have

$$
c_{p}^{(p)}=\frac{(-1)^{p-1}}{p\left(\begin{array}{c}
2 p \\
p
\end{array}\right)}
$$

and the the leading coefficient of $T_{p}(x)$ is $2^{p-1}$. It follows that

$$
d_{p}=\frac{2^{p}}{\left(\begin{array}{c}
2 p \\
p
\end{array}\right)}
$$

and the lemma is proven. 


\section{Appendix B. Proof of Theorem 3}

Proof. Recall the form of the basis functions,

$$
\phi_{j}(\xi)=2 \sin ((p+j) \xi)+2 \sum_{k=1}^{p} \alpha_{k}^{(j)} \sin (k \xi) .
$$

Note first that $\phi_{j}(\xi)$ is a trigonometric polynomial. Thus the number of zeros of any non-trivial combination, $\psi(\xi, \mathbf{a})$, of these functions is finite in any continuous bounded interval.

Let $x=\cos (\xi)$ and consider the function

$$
f^{(p)}(x, \mathbf{a})=1-2 \sum_{k=1}^{p+n} a_{k} k T_{k}(x)
$$

where $T_{k}(x)$ is the $k^{\text {th }}$ Chebyshev polynomial, and the coefficients $a_{k}$ are consistent with a central difference scheme of order $\mathcal{O}\left(\Delta x^{2 p}\right)$. As before we let $\mathbf{a}=\left(a_{p+1}, \ldots, a_{p+n}\right)$. Note that $f^{(p)}$ depends linearly on $\mathbf{a}$.

By the same argument as in Lemma $1, f^{(p)}(x, \mathbf{a})$ is a polynomial in $x$ of degree $p+n$ with a root of multiplicity $p$ at $x=1$. We may thus write

$$
f^{(p)}(x, \mathbf{a})=(1-x)^{p} \mathcal{P}_{n}(x, \mathbf{a}),
$$

where $\mathcal{P}_{n}(x, \mathbf{a})$ is some polynomial of degree $n$ that depends linearly on the elements in $\mathbf{a}$.

Just like in Theorem 2 the error function satisfies

$$
\frac{\partial E(\xi, \mathbf{a})}{\partial \xi}=f^{(p)}(\cos (\xi), \mathbf{a})=(1-\cos (\xi))^{p} \mathcal{P}_{n}(\cos (\xi), \mathbf{a}) .
$$

Thus, $E(\xi, \mathbf{a})$ has an extreme point at $\xi=0$ and at most $n$ other extreme points $\xi_{1} \leq \xi_{2} \leq \cdots \leq \xi_{n}$ in $\left[0, \xi_{\max }\right]$. If $\xi_{1}=0$ the stencil would be of higher order than $\mathcal{O}\left(\Delta x^{2 p}\right)$ according to Theorem 2 , so we may assume that $0<\xi_{1}$.

Consider now another error function, $E(\xi, \mathbf{b})$. Linearity gives

$$
\begin{aligned}
\frac{\partial(E(\xi, \mathbf{a})-E(\xi, \mathbf{b}))}{\partial \xi} & =(1-\cos (\xi))^{p}\left[\mathcal{P}_{n}(\cos (\xi), \mathbf{a})-\mathcal{P}_{n}(\cos (\xi), \mathbf{b})\right] \\
& =(1-\cos (\xi))^{p} \mathcal{P}_{n}(\cos (\xi), \mathbf{a}-\mathbf{b}) .
\end{aligned}
$$


but also

$$
\begin{aligned}
\frac{\partial(E(\xi, \mathbf{a})-E(\xi, \mathbf{b}))}{\partial \xi} & =\frac{\partial}{\partial \xi}\left[\left(E_{c}(\xi)-\psi(\xi, \mathbf{a})\right)-\left(E_{c}(\xi)-\psi(\xi, \mathbf{b})\right)\right] \\
& =\frac{\partial}{\partial \xi} \psi(\xi, \mathbf{b}-\mathbf{a})
\end{aligned}
$$

where, as before, $\psi$ is a linear combination of the basis functions, $\left\{\phi_{j}\right\}, j=$ $1, \ldots, n$. The vectors $\mathbf{a}$ and $\mathbf{b}$ are completely general so we may write $\mathbf{c}=$ $\mathbf{b}-\mathbf{a}$ for any vector $\mathbf{c} \in \mathbb{R}^{n}$. Thus $\psi(\xi, \mathbf{c})$ has at most $n$ extrema in the open interval $\left(0, \xi_{\max }\right]$. Obviously there is at most one zero in between any two consecutive extreme points. Thus $\psi(\xi, \mathbf{c})$ has at most $(n-1)$ zeros in $\left(0, \xi_{\max }\right]$. This completes the proof. 


\section{Appendix C. Stencil coefficients}

\begin{tabular}{cccccc} 
& $\operatorname{DRP}(4,7)$ & $\operatorname{BB}(4,9)$ & $\mathrm{ZLJ}(2,7)$ & $\operatorname{Remez}(2,7)$ & $\operatorname{Remez}(2,9)$ \\
\hline a1 & 0.76868543 & 0.84157013 & 0.75996130 & 0.78028389 & 0.82535056 \\
$\mathrm{a} 2$ & -0.16494834 & -0.24467863 & -0.15812200 & -0.17585010 & -0.22669661 \\
a3 & 0.02040375 & 0.05946358 & 0.01876090 & 0.02380544 & 0.05059599 \\
a4 & & -0.00765090 & & & -0.00593632 \\
d0 & & & 0.1 & \\
d1 & & & -0.07638461 & & \\
d2 & & 0.03228961 & & \\
d3 & & -0.00590499 & &
\end{tabular}

Table C.2: Stencil coefficients. Here $a_{k}$ corresponds to advective terms while $d_{k}$ corresponds to dissipative terms. 


\section{References}

[1] B. Auld, Acounstic Fields and Waves in Solids, Wiley-Interscience, New York, 1973.

[2] L. Brillouin, Wave Propagation in Periodic Structures, Dover, New York, 1953.

[3] L. Brillouin, Wave Propagation and Group Velocity, Academic Press, New York, 1960.

[4] F. Jenkins, H. White, Fundamentals of Physical Optics, McGraw-Hill, New York, 1937.

[5] J. Lighthill, Waves in Fluids, Cambridge University Press, Cambridge, 1978.

[6] G. B. Whitham, Linear and Nonlinear Waves, Wiley-Interscience, 1974.

[7] L. N. Trefethen, Group velocity in finite difference schemes, SIAM review 24 (1982) 113-136.

[8] L. N. Trefethen, Group velocity interpretation of the stability theory of Gustafsson, Kreiss, and Sundström, Journal of Computational Physics 49 (1983) 199-217.

[9] S. Davis, Low-dispersion finite difference methods for acoustic waves in a pipe, J. Acoustic Soc. Amer. 90 (1991) 2775-2781.

[10] D. Gottlieb, E. Turkel, Dissipative two-four methods for time-dependent problems, Math. Comp. 29 (1976) 703-723.

[11] S. K. Lele, Compact finite difference schemes with spectral-like resolution, Journal of Computational Physics 103 (1992) 16-42.

[12] Z. Haras, S. Ta'asan, Finite-difference schemes for long-time integration, Journal of Computational Physics 114 (1994) 265-279.

[13] C. Tam, J. Webb, Dispersion-reltion-preserving finite difference schemes for computational acoustics, Journal of Computational Physics 107 (1993) 262-281. 
[14] D. W. Zingg, H. Lomax, H. Jurgens, An optimized finite-difference scheme for wave propagation problems, AIAA paper 93(0459) (1993).

[15] D. W. Zingg, H. Lomax, H. Jurgens, High-accuracy finite-difference schemes for linear wave propagation, SIAM J. Sci. Comput. 17 (1996) 328-346.

[16] C. Bogey, C. Bailly, A family of low dispersive and low dissipative explicit schemes for flow and noise computations, Journal of Computational Physics 194 (2004) 194-214.

[17] C. Lui, S. K. Lele, Direct numerical simulation of spatially developing, compressible, turbulent mixing layers, AIAA-Paper 2001-0291 (2001).

[18] R. Hixon, Prefactored small-stencil compact schemes, Journal of Computational Physics 165 (2000) 522-541.

[19] R. Hixon, E. Turkel, Compact implicit maccormac-type schemes with high accuracy, Journal of Computational Physics 158 (2000) 51-70.

[20] S. Jakobsson, Frequency optimized computation methods, Journal of Scientific Computing 26 (2006) 329-362.

[21] J. W. Nehrbass, J. O. Jevtić, R. Lee, Reducing the phase error for finitedifference methods without increasing the order, IEEE Trans. Antennas and Propagation 46 (1998) 1194-1201.

[22] C. David, P. Sagaut, Drp scheme optimization, arXiv:math/0611846 (2008).

[23] D. W. Zingg, Comparison of high-accuracy finite-difference methods for linear wave propagation, SIAM J. Sci. Comput. 22 (2000) 476-502.

[24] G. A. Watson, Approximation Theory and Numerical Methods, John Wiley \& Sons Ltd., 1980.

[25] J. W. Young, General theory of approximation by functions involving a given number of arbitrary parameters, Trans. A.M.S. 8 (1907) 331-344.

[26] J. Li, General explicit difference formulas for numerical differentiation, Journal of Computational and Applied Mathematics 183 (2005) 29-42. 
[27] E. Remez, Sur un procédé convergent d'approximations successives pour déderminer les polynômes d'approximation, Comptes Rendues 198 (1934) 2063-2065.

[28] E. Remez, Sur le calcul effectif des polynomes d'approximation de tchebichef, Comptes Rendues 199 (1934) 337-340.

[29] E. Novodvorski, I. Pinsker, On a process of equilization of maxima, Uspehi Mat. Nauk. 6 (1951) 174-181.

[30] L. Veidinger, On the numerical determination of the best approximations in the Chebyshev sense, Numer. Math 2 (1960) 95-105.

[31] M. Svärd, J. Nordström, Review of summation-by-parts schemes for initial-boundary-value problems, Journal of Computational Physics 268 (2014) 17-38.

[32] D. C. D. R. Fernández, J. E. Hicken, D. W. Zingg, Review of summationby-parts operators with simultaneous approximation terms for the numerical solution of partial differential equations, Computers \& Fluids 95 (1994) 171-196.

[33] G. Erlebacher, M. Y. Hussaini, C. W. Shu, Interaction of a shock with a longitudinal vortex, J. Fluid Mech. 337 (1997) 129-153. 